\title{
HIRA contributes to zygote formation in mice and is implicated in human 1PN zygote phenotype
}

\author{
Rowena Smith, Sue J Pickering ${ }^{2}$, Anna Kopakaki², K J Thong², Richard A Anderson ${ }^{1,2}$ and \\ Chih-Jen $\operatorname{Lin}^{1}$ \\ ${ }^{1}$ MRC Centre for Reproductive Health, University of Edinburgh, Queen's Medical Research Institute, Edinburgh \\ Bioquarter, Edinburgh, UK and 'Edinburgh Fertility and Reproductive Endocrine Centre, Simpson's Centre for \\ Reproductive Health, Edinburgh Royal Infirmary, Edinburgh, UK
}

Correspondence should be addressed to C-J Lin; Email: Chih-Jen.Lin@ed.ac.uk

\begin{abstract}
Elucidating the mechanisms underpinning fertilisation is essential to optimising IVF procedures. One of the critical steps involves paternal chromatin reprogramming, in which compacted sperm chromatin packed by protamines is removed by oocyte factors and new histones, including histone $\mathrm{H} 3.3$, are incorporated. HIRA is the main $\mathrm{H} 3.3$ chaperone governing this protamine-to-histone exchange. Failure of this step results in abnormally fertilised zygotes containing only one pronucleus (1PN), in contrast to normal two-pronuclei (2PN) zygotes. 1PN zygotes are frequently observed in IVF treatments, but the genotype-phenotype correlation remains elusive. We investigated the maternal functions of two other molecules of the HIRA complex, Cabin1 and Ubn1, in mouse. Loss-of-function Cabin1 and Ubn1 mouse models were developed: their zygotes displayed an abnormal 1PN zygote phenotype. We then studied human 1PN zygotes and found that the HIRA complex was absent in 1PN zygotes that lacked the male pronucleus. This shows that the role of the HIRA complex in male pronucleus formation potentially has coherence from mice to humans. Furthermore, rescue experiments in mouse showed that the abnormal 1PN phenotype derived from Hira mutants could be resolved by overexpression of HIRA. We have demonstrated that HIRA complex regulates male pronucleus formation in mice and is implicated in humans, that both CABIN1 and UBN1 components of the HIRA complex are equally essential for male pronucleus formation, and that rescue is feasible.

Reproduction (2021) 161 697-707
\end{abstract}

\section{Introduction}

Fertilisation of an oocyte by a sperm to produce a zygote and then an embryo underlies mammalian reproduction. As a treatment for infertility, IVF is well established, but although fertilisation remains at its centre, our understanding of this process is limited, preventing the possibility of specific diagnoses and thus treatments that may be relevant to some couples, and which may improve success rates overall. To achieve a successful fertilisation event, consequential steps including oocyte activation, chromatin reprogramming of the sperm (protamine-to-histone exchange and histone reassembly), and formation of the pronuclei are essential. These all rely on the quality of the oocytes, and this is dependent on oocyte stored factors (Gosden 1996, Swain \& Pool 2008). Therefore, it is imperative to elucidate the underlining regulatory mechanisms involved in these steps, in the search for potential new IVF treatments which can overcome defects found in poor quality oocytes. An intriguing clinical phenotype arising from abnormal fertilisation is the one-pronucleus
(1PN) zygote. It is observed after overnight culture following both conventional IVF and ICSI and occurs in $3-17 \%$ of fertilised oocytes, though mostly in the range of 4-8\% (Azevedo et al. 2014, Si et al. 2019). However, the aetiology is unknown.

The building blocks of chromatin are the core histones $\mathrm{H} 2 \mathrm{~A}, \mathrm{H} 2 \mathrm{~B}, \mathrm{H} 3$, and $\mathrm{H} 4$. In addition to these canonical histones, there are variants which share similar sequences with canonical histones and which carry out diverse functions. One example is histone variant $\mathrm{H} 3.3$, a version of $\mathrm{H} 3$ with wider regulatory roles, one of which, significantly, is that it allows $\mathrm{H} 3.3$ to bind to chromatin independently of the cell cycle (Maze et al. 2014). The incorporation of histones onto chromatins is tightly regulated by histone chaperones (Buschbeck \& Hake 2017, Hammond et al. 2017). HIRA complex, which comprises HIRA, CABIN1, and UBN1, is one of the H3.3 chaperones (Rai et al. 2011).

The role of H3.3 incorporation during fertilisation and the underlying mechanism of mediation by histone chaperone HIRA, have been reported in model organisms such as drosophila (Loppin et al. 2005) and carp 
(Zhao et al. 2011). However, direct evidence in mouse had not been reported until a few years ago (Inoue \& Zhang 2014, Lin et al. 2014, Tang et al. 2015). Applying a mouse genetic model, we reported that maternal Hira is essential for the completion of chromatin reprogramming of the sperm during fertilisation; mutant Hira oocytes formed abnormal $1 \mathrm{PN}$ zygotes that lacked a male pronucleus due to the failure of $\mathrm{H} 3.3$ incorporation (Lin et al. 2014). However, the maternal roles of the other molecules in the Hira complex, Cabin 1 and Ubn 1, remain unknown in mammals.

In this study, we explored the maternal role of the remaining candidates within the HIRA complex, Ubn 1 and Cabin 1, in the mouse. We have found that they are essential in the paternal chromatin reprogramming event. Loss-of-function mouse models for either Ubn 1 or Cabin 1 exhibited the same 1PN phenotypes as Hira mutants, and rescue experiments revealed that the abnormal 1PN phenotype of Hira mutants could be restored back to $2 \mathrm{PN}$ zygotes.

Our examination of abnormal human 1PN zygotes showed that failure of HIRA complex binding is possibly involved in the fertilisation cascade steps similar to the mouse.

\section{Materials and methods Animals}

Mouse experiments were approved by the University of Edinburgh's Animal Welfare and Ethical Review Board (AWERB) and carried out under the authority of a UK Home Office Project Licence. The mouse lines used in this study carried conditional floxed alleles for Cabin 1 and for Hira on C57BL/6 backgrounds (both were provided from Prof Peter Adams previously of the Beatson Institute, UK, now at Sanford Burnham Prebys, USA). Floxed Cabin 1 (Cabin $1 \mathrm{fl} / \mathrm{fl}$ ) and floxed Hira (Hira fl/fl) mouse lines were crossed with a Zp3-Cre mouse line ((de Vries et al. 2000); provided from Prof Petra Hajkova, Imperial College, London) that expresses Cre recombinase in the female germline to generate heterozygous and homozygous mutant oocytes.

\section{Manipulation of oocytes and embryos}

Female mice were primed by administration of 7.5 I.U. pregnant mare serum gonadotrophin (PMSG from Prospec Protein Specialists, USA) or $80 \mu \mathrm{L}$ ultra-superovulation reagent (CARD HyperOva Cosmo Bio, Japan; (Hasegawa et al. 2016)) and $48 \mathrm{~h}$ later fully grown oocytes were isolated. For in vitro maturation (IVM), oocytes were cultured in M16 medium (Sigma) at $37^{\circ} \mathrm{C}, 6 \% \mathrm{CO}_{2}$ and $5 \% \mathrm{O}_{2}$ for 18 to $24 \mathrm{~h}$.

For the zygote collection, $48 \mathrm{~h}$ after PMSG priming, 7.5 I.U. human chorionic gonadotrophin (hCG, Chorulon $\AA$ from Intervet) was injected and the mice mated with $\mathrm{C} 57 \mathrm{BL} / 6$ males. The following day zygotes were collected from the oviducts and cumulus cells were removed after treatment with $300 \mu \mathrm{g} /$ $\mathrm{mL}$ hyaluronidase (Millipore).

The microinjection procedure was the same as previously described (Chen et al. 2013, Lin et al. 2014). The micromanipulation platform was equipped with a microinjector (Femtolet 4i, Eppendorf), an inverted microscope (Leica, DMi8), and micromanipulators (Narishige). For Ubn 1 knockdown experiments, oocytes were microinjected with $2 \mathrm{mM}$ of either control or Ubn 1 antisense morpholino oligos (Gene Tools). Injected oocytes were cultured in M16 for subsequential in vitro maturation and in vitro fertilisation experiments. For validation of knock-down by immunofluorescence, oocytes were cultured overnight in M16+IBMX to retain the GV stage.

For the HIRA rescue experiments, Hira-GFP (a gift from D Dutta) and GFP (a gift from M Anger, IAPG, CAS) were used as templates for in vitro synthesis of RNA using mMessage

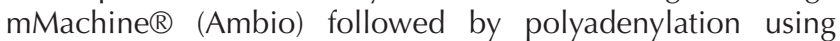
a Poly(A) tailing kit (Applied Biosystems). Transcripts were purified by phenol:chloroform extraction and isopropanol precipitation and diluted to $160 \mathrm{ng} / \mu \mathrm{L}$ for microinjection.

In vitro fertilisation (IVF) was performed as previously described (Sztein et al. 2000) with minor modifications. Briefly, sperm were collected from the vasa deferentia and then capacitated for $1.5 \mathrm{~h}$ in human tubal fluid (HTF) medium (Millipore). Sperm cells were then incubated with intact cumulus-oocyte complexes (final sperm concentration $\sim 1.4 \times 10^{5} / \mathrm{mL}$ ) or zona-free oocytes (for rescue experiments; final sperm concentration $\sim 3.1 \times 10^{5} / \mathrm{mL}$ ) for $5 \mathrm{~h}$. After removing excess sperm and cumulus lysate, presumptive fertilized eggs were transferred to equilibrated KSOM+AA medium (Millipore) for further culture and analysis. In order to facilitate the IVF efficiency and pronuclear formation of our $\mathrm{C} 57 \mathrm{BL} / 6$ inbred strain, we adapted the culture conditions by using low oxygen concentrations, similar to Sztein et al. (2000). Specifically, we used a benchtop incubator for IVF and embryo culture with $6 \% \mathrm{CO}_{2}, 5 \% \mathrm{O}_{2}$, and $\mathrm{N}_{2}$.

\section{Immunohistochemistry (IHC)}

IHC was carried out according to standard protocols. Briefly, ovaries from 4 to 8 -week-old mice were fixed in $4 \%$ paraformaldehyde overnight at $4{ }^{\circ} \mathrm{C}$ then washed for $3 \times 10$ min in PBS followed by transfer to $70 \%$ ethanol before being processed into paraffin wax blocks. Sections of $5 \mu \mathrm{m}$ were cut and mounted onto SuperfrostPlus slides (Thermo Fisher Scientific). After de-waxing and rehydration, slides were subject to antigen retrieval by pressure cooking at maximum pressure for $5 \mathrm{~min}$ in $0.01 \mathrm{M}$ sodium citrate buffer $\mathrm{pH} 6$.

To reduce non-specific staining from endogenous peroxidases, sections were incubated in 3\% hydrogen peroxide (Sigma) solution for $15 \mathrm{~min}$.

Prior to incubation with the primary antibody, incubation with normal serum and BSA was carried out to reduce nonspecific binding. The brown signal was revealed after incubation with ImmPRESSTM HRP Anti Rabbit IgG (Peroxidase) Polymer detection kit followed by ImmPACTTM DAB Peroxidase (HRP) substrate (both from Vector laboratories). 
The sections were counterstained with haematoxylin and imaged on a Zeiss Axiolmager Z1.

\section{Immunofluorescence (IF)}

IF experiments were performed as previously reported (Lin et al. 2014). Most oocytes/embryos were directly fixed in 4\% paraformaldehyde for $15 \mathrm{~min}$. To remove unbound histones, some oocytes were incubated with pre-extraction buffer containing $0.5 \%$ Triton X-100 for 5 min, following Hajkova and Nashun protocol (Paull et al. 2013), before being fixed in $4 \%$ paraformaldehyde. Fixed samples were permeabilised by $0.5 \%$ Triton X-100 and then blocked by $5 \%$ BSA before incubation with primary antibodies. The antibodies used were HIRA (LSBio LS-C137477), UBN1 (a gift from Peter Adams), H3.3 (Abnova H00003021-M01), CABIN1 (Abcam ab3349), H3K9me2 (Abcam ab1220), H3K9me3 (Abcam ab8898). Secondary antibodies used were donkey anti rabbit IgG, 568 nm (Thermo Fisher A10042), and donkey anti mouse IgG, 488 $\mathrm{nm}$ (Thermo Fisher A21202).

After washing and counterstaining with DAPI (Life Technologies D3571), samples were mounted using

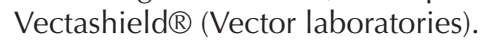

Images of stained oocytes/embryos were acquired by a spinning disk confocal (CSU-W1, Yokogawa) on an upright microscope frame (BX-63, Olympus) using a 30 or $60 \times$ silicon oil immersion objective (UPLSAPO 60XS2, Olympus) (Percharde et al. 2018). IF signal intensity was quantified using Fiji software.

\section{Proximity ligation assay (PLA)}

Proximity ligation assay was performed according to the

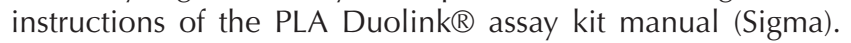
Briefly, WT zygotes (2- and 4-h post IVF) were fixed for 15 min in $4 \%$ paraformaldehyde followed by permeabilisation for $20 \mathrm{~min}$ in $0.5 \%$ Triton X-100 in PBS. The samples were then blocked in PLA Duolink® blocking solution before being incubated with primary antibodies: either mouse anti HIRA (LSBio LS-C137477) and rabbit anti UBN1 (a gift from Peter Adams) or mouse anti HIRA and rabbit anti CABIN1 (Abcam ab3349). As a negative control the antibodies raised in rabbit were incubated with mouse anti hCG (Abcam ab9582) which should not be present in mouse tissues.

All wash steps were with wash buffer A. After washing, the samples were incubated with the PLA PLUS and MINUS probes, diluted as directed, for $60 \mathrm{~min}$ at $37^{\circ} \mathrm{C}$. Following washing, the samples were incubated for $30 \mathrm{~min}$ at $37^{\circ} \mathrm{C}$ in ligation reaction mixture followed by further washes before being incubated for $100 \mathrm{~min}$ at $37^{\circ} \mathrm{C}$ in the reaction mixture for rolling circle amplification. The samples were mounted on SuperfrostPlus ${ }^{\circledR}$ slides (Thermo Fisher Scientific) using Duolink $₫$ mounting medium plus DAPI. Images were acquired by a spinning disk confocal (CSU-W1, Yokogawa) on an upright microscope frame (BX-63, Olympus) using a 30 or $60 \times$ silicon oil immersion objective (UPLSAPO 60XS2, Olympus). Quantification of interaction foci was performed using Fiji software.

\section{Human one-pronucleus zygotes}

Approvals were obtained from an ethical committee (East of Scotland Research Ethics Service; REC 16/ES/0039) and the Human Fertilisation and Embryology Authority (HFEA), research licence (R0204), for the collection of human onepronucleus (1PN) zygotes, in collaboration with the Edinburgh Fertility and Reproductive Endocrine Centre (EFREC). Patients were recruited before oocyte collection, and samples were collected from couples who gave informed consent, in writing.

Oocyte and sperm collection, fertilisation by IVF or ICSI, and embryo culture procedures, were performed following the routine operational protocols of EFREC and described in Sciorio et al. (Sciorio et al. 2018). Briefly, cumulus-oocytecomplexes (COC) were retrieved from follicular fluid, and then oocytes were isolated and washed. Oocytes were inseminated by IVF or ICSI as clinically indicated. Inseminated oocytes were cultured in G-IVF Plus medium (Vitrolife, Sweden) at $37^{\circ} \mathrm{C}$ and $6 \% \mathrm{CO}_{2}$ in atmospheric air. Single pronucleus zygotes were identified approximately $18 \mathrm{~h}$ post IVF or ICSI.

Embryo disposal, transfer, and witness documents followed the regulations of the HFEA clinic licence (0201). 1PN zygotes were individually labelled and transported using a portable incubator in G-MOPS Plus medium (Vitrolife, Sweden) at $37^{\circ} \mathrm{C}$ from the clinic laboratory to the research laboratory. Zygotes were then fixed in 4\% paraformaldehyde for $15 \mathrm{~min}$ at room temperature. IF procedure and confocal imaging were the same as described above.

Antibody against $\mathrm{H} 3 \mathrm{~K} 9 \mathrm{me} 3$ was used as a marker of human female chromatin (van de Werken et al. 2014) and UBN1, HIRA, H3.3, and CABIN1 antibodies were also applied.

\section{Statistical analyses}

For analyses of the proportion of pronuclear formation, the $\chi^{2}$ test was used. For analyses of IF intensity, two-tailed $t$-test with unequal variance was used. ANOVA was used for PLA quantification. All error bars indicate S.D..

\section{Results}

\section{Maternal HIRA complex is involved in male chromatin deposition in mouse oocytes}

First, we examined whether the constituent molecules of the HIRA complex were present in WT mouse oocytes during oogenesis. Immunohistochemistry of mouse ovarian sections demonstrated that both CABIN1 and UBN1 were present in oocytes and were particularly enriched in germinal vesicle (GV) nuclei (Fig. 1A). Wholemount immunofluorescence (IF) of GV stage oocytes also confirmed this observation (Fig. 1A). Therefore, both CABIN1 and UBN1 are oocyte factors that accumulate in GV oocytes.

We postulated that both CABIN1 and UBN1 collaborate with HIRA and are involved in the chromatin reprogramming processes during fertilisation. We collected and fixed newly fertilised IVF embryos (2-4 h post fertilisation, PN 0 stage) 

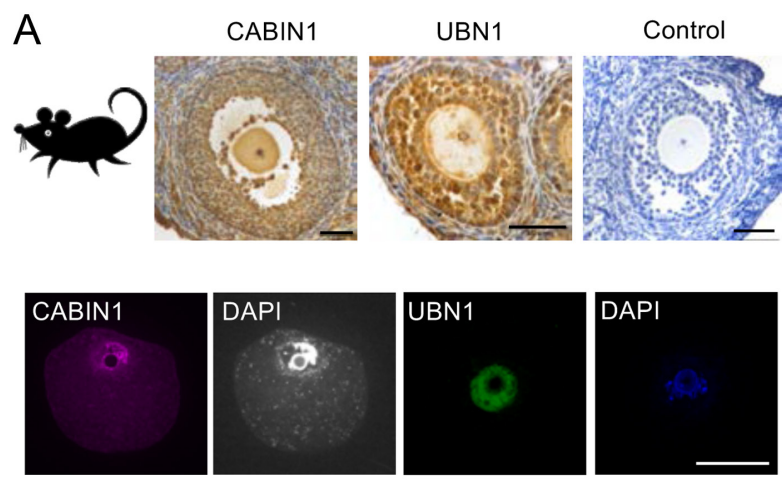

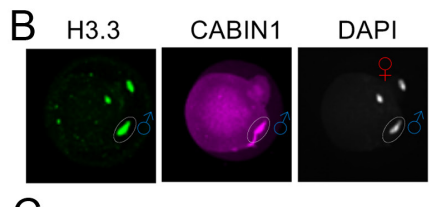

.

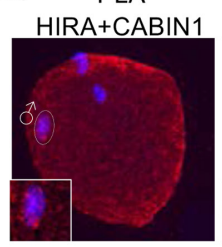

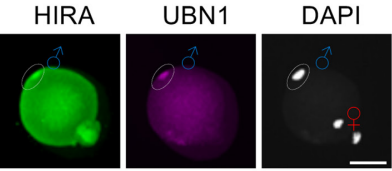

PLA

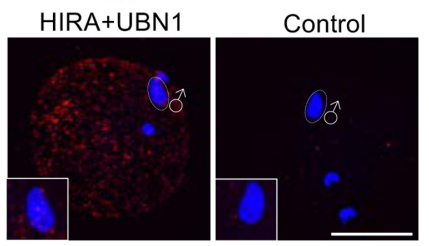

Figure 1 Maternal HIRA complex associates with male chromatin after fertilisation. (A) CABIN1 and UBN1 are maternal factors present in the mouse oocytes. Immunohistochemisty of murine ovarian sections (upper panel) and immunofluorescence of GV oocytes (lower panel) showed positive signals of CABIN1 and UBN1 in the GV nuclei. Scale bar $=50 \mu \mathrm{m}$. (B) CABIN1 and UBN1 bind to male chromatin after fertilisation. Immunofluorescence of $\mathrm{H} 3.3$ and CABIN1 (left panel) and HIRA and UBN1 (right panel) show positive signals in the decondensed male chromatin after fertilisation. Scale bar $=50 \mu \mathrm{m}$. (C) Proximity ligation assay (PLA) confirmed the interactions of CABIN1 with HIRA (left panel) and UBN1 with HIRA (right panel) in the male chromatin during sperm decondensation. Left panel: images of embryos stained with antibodies against HIRA and CABIN1. Negative control: antibody of hCG and CABIN1; Right panel: images of embryos stained with antibodies against HIRA and UBN1. Negative control: antibody of hCG and UBN1; Inset: enlarged (1.7-fold) images of male chromatins. Scale bar=50 $\mu$ m.

in which female chromatin had completed the 2 nd meiotic division and in doing so had segregated sister chromatids. Meanwhile, male chromatin had undergone DNA decondensation. IF showed that CABIN1 had deposited into chromatin and co-localised with H3.3. UBN1 had also deposited into male chromatin and showed a similar pattern to HIRA (Fig. 1B; (Lin et al. 2014)).

To provide further evidence of the interactions within HIRA complex during male chromatin decondensation, we performed proximity ligation assay (PLA), a sensitive assay for visualisation of proteinprotein interactions. First, we examined the interaction of UBN1 with HIRA and CABIN1 with HIRA in the GV oocytes, respectively. We detected significantly more positive PLA signals in the nuclei of both UBN1 with HIRA and CABIN1 with HIRA groups than either UBN1 or CABIN1 alone or with hCG (a protein which is absent in the mouse) antibody (Supplementary Fig. 1 , see section on supplementary materials given at the end of this article). Next, we examined the PNO stage zygotes and detected positive signal foci for HIRA and CABIN1 and HIRA and UBN1 in the decondensed male chromatin, but not in the negative controls (Fig. 1C). We also observed foci for HIRA and CABIN1 as well as HIRA and UBN1 distributed throughout the zygotes. It suggests that CABIN1 and UBN1 potentially interact in the cytoplasm and contribute to oocyte developmental competence. As we previously reported, HIRA has a dual role for impacting on male pronuclear formation and zygotic genome activation (Lin et al. 2014) and (Smith et al. 2020). Thus, we demonstrated that both CABIN1 and UBN1 are maternal factors deposited in oocytes and associate with decondensed male chromatin after fertilisation and that they physically interact with HIRA. We hypothesised that the molecules within the HIRA complex could collaboratively play a role during paternal chromatin reprogramming.

\section{HIRA complex is essential for male pronucleus formation during fertilisation in mice}

We then generated three new HIRA complex models, each one with oocyte loss-of-function in one component part of the HIRA complex (Supplementary Fig. 2A).

We generated a new Hira conditional knockout mouse line different from our previous report (Lin et al. 2014). We used a Zp3-Cre mouse line to inactivate the Hira flox/flox alleles and so delete exon 6-7. We validated the loss of Hira mRNA in the GV oocytes by qRT-PCR and the significant reduction of HIRA in both GV oocytes and zygotes confirmed by IF which were similar to our previous report (Lin et al. 2014; data not shown). In addition, we observed an infertile phenotype in the new Hira mutant mice which was consistent with that seen in our previous model (no pups were generated from four mutant females mated with proven stud males for over 4 months; compared with an average litter size of six from heterozygous control; data not shown). Zygotes retrieved from this new Hira mutant also displayed 1PN phenotypes due to the failure of male pronucleus formation (73.5\%; Fig. 2A).

For Ubn 1, we applied the morpholino antisense oligos knockdown approach to oocytes ((Lin et al. 2013, 2014); Supplementary Fig. 2B). After injection of morpholino oligos against Ubn1 (Ubn1 MO), the level of UBN1 in oocytes was significantly reduced compared to control oligos (Ctrl MO) (Supplementary Fig. 2C; 49\% reduction of UBN1 after knockdown). Interestingly, fertilised Ubn1 MO matured oocytes revealed a high proportion $(87 \%)$ of abnormal 1PN zygotes (Fig. 2B). To examine whether $\mathrm{H} 3.3$ can incorporate into male chromatin in 

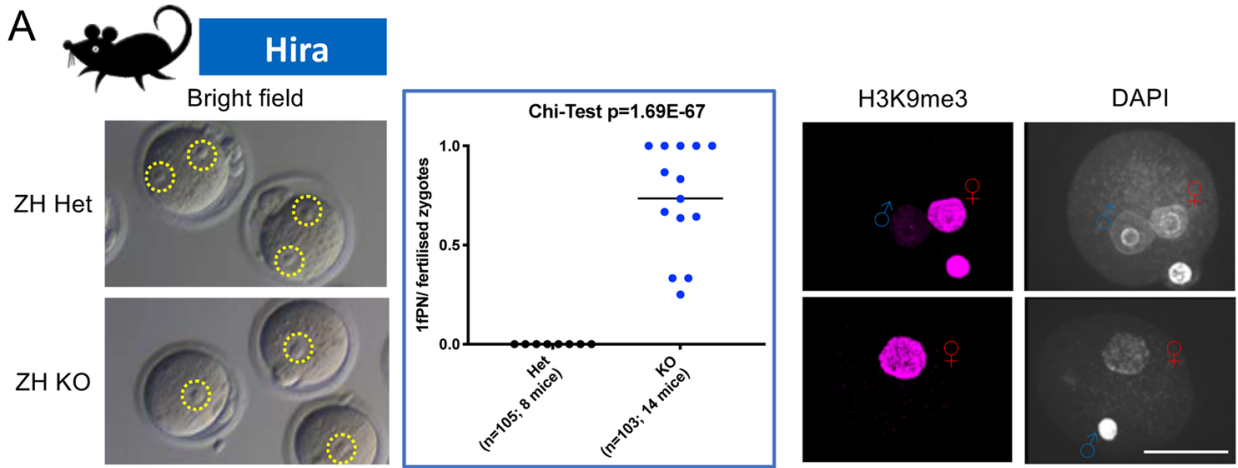

$\mathrm{B}$

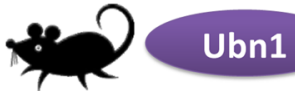

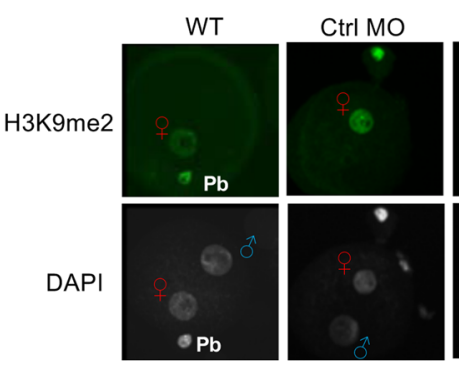

D

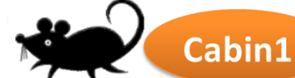

Bright field

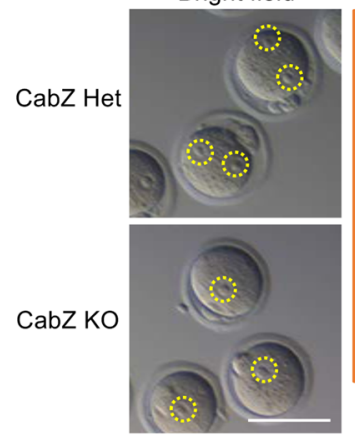

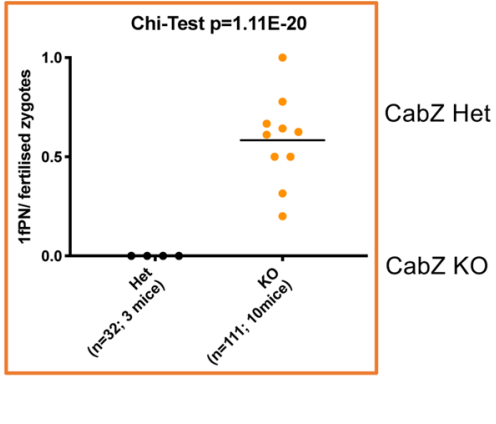

C
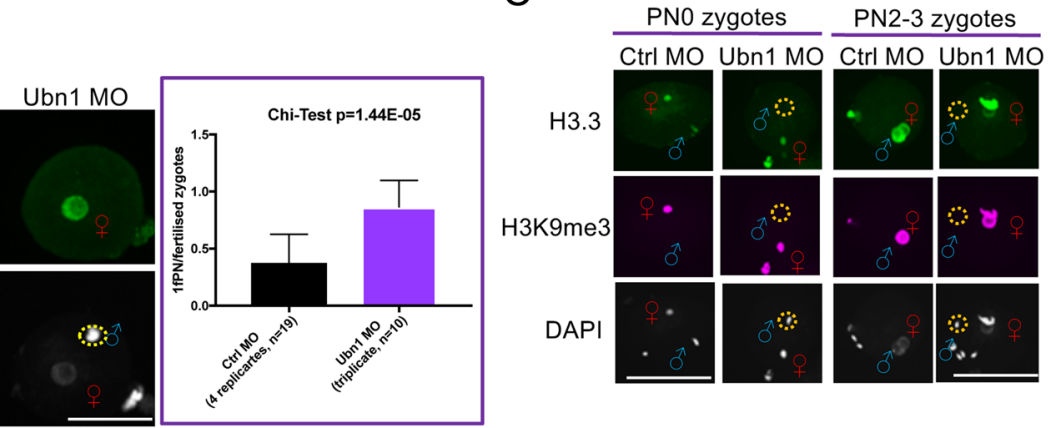

E
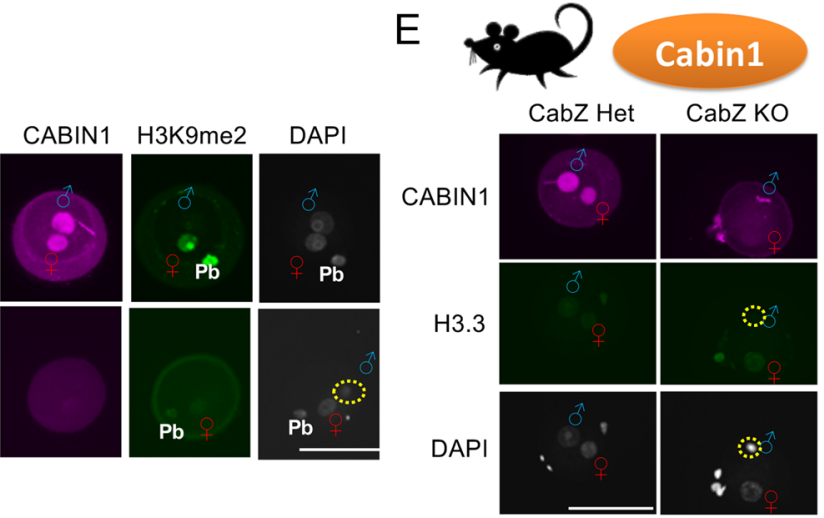

Figure 2 Loss-of-function maternal HIRA complex in mice gave rise to abnormal one-pronucleus (1PN) zygotes due to failure to form male pronuclei. (A) Hira mutant oocytes formed abnormal 1PN zygotes after fertilisation. Brightfield images showed abnormal 1PN zygotes of Hira mutants ( $\mathrm{HH} \mathrm{KO}$ ), in contrast to normal two-pronuclei (2PN) zygotes in the controls (ZH Het; left panel). Abnormal $1 \mathrm{PN}$ formation rate was increased in the Hira mutant zygotes (middle panel). Immunofluorescence showed that male pronucleus formation is impaired in Hira mutant zygote (right panel). Female chromatin was labelled with histone H3K9me3. Scale bar $=40 \mu \mathrm{m}$. (B) Ubn 1 knockdown oocytes formed $1 \mathrm{PN}$ zygotes after fertilisation. Male pronucleus formation is impaired in Ubn 1 morpholino $(\mathrm{MO})$ knocked down zygotes. Histone H3K9me2 stained for female chromatin (left panel). Abnormal 1PN formation rate was increased in the Ubn1 MO knockdown zygotes (right panel). Scale bar=40 $\mu \mathrm{m}$. (C) Histone H3.3 failed to incorporate into male chromatin in the Ubn1 knockdown zygotes. Left panel: in Ubn 1 knockdown embryos immediately after fertilisation (pronuclear stage 0, PNO), H3.3 failed to incorporate into male chromatin which was undergoing the decondensation process. Right panel: H3.3 failed to incorporate into male chromatin in Ubn 1 knockdown zygotes at PN2-3 stage. Histone $\mathrm{H} 3 \mathrm{~K} 9 \mathrm{me} 2$ stained for female chromatin. Scale bar $=80 \mu \mathrm{m}$. (D) Cabin 1 mutant oocytes formed abnormal 1PN zygotes after fertilisation. Brightfield images showed abnormal 1PN zygotes formed in the Cabin 1 mutants (CabZ KO), in contrast to normal 2PN zygotes in the controls (CabZ Het; left panel). Abnormal 1PN formation rate was increased in the Cabin 1 mutant zygotes (middle panel). Immunofluorescence showed that male pronucleus formation is impaired in the Cabin 1 mutants. Female chromatin was labelled with histone H3K9me2 (right panel). Scale bar $=80 \mu \mathrm{m}$. (E) Histone H3.3 failed to incorporate into male chromatin in the Cabin 1 mutant zygotes. Scale bar $=80 \mu \mathrm{m}$. 
Ubn 1 KD zygotes, we performed IF on PN0 and PN2-3 stages zygotes, respectively. After fertilisation, H3.3 was incapable of incorporating into male chromatin during the sperm decondensation process ( $\mathrm{PNO}$ stage) in Ubn $1 \mathrm{KD}$ zygotes. Later, it fails to incorporate into the male pronucleus (PN2-3 stage). In contrast, H3.3 incorporated into male chromatin in both $\mathrm{PNO}$ and PN2-3 stage zygotes of controls (Ctrl MO) (Fig. 2C).

To investigate the maternal role of Cabin1, we again used the Zp3-Cre approach and crossed to Cabin 1 flox/ flox mice to delete exon 6 (Supplementary Fig. 2A). Cabin 1 mutant mice (CabZ KO) also showed an infertile phenotype, and fertilised CabZ KO zygotes revealed the abnormal 1PN phenotype due to the failure of male pronucleus formation (Fig. 2D). IF on CABIN1 zygotes demonstrated that $\mathrm{H} 3.3$ was incapable of incorporating into male chromatin in CabZ KO oocytes. In contrast, H3.3 incorporated into pronuclei of Cabin 1 heterozygous controls (CabZ Het) (Fig. 2E).

Since UBN1 has been reported as providing the specificity of H3.3 binding (Ricketts et al. 2015) and CABIN1 functions as enhancing and stabilising the overall HIRA complex (Ray-Gallet et al. 2018), we also investigated whether the loss-of-function of Ubn 1 and Cabin 1 compromised the overall stability of the HIRA complex in the oocytes. We performed IF on Ubn 1 knockdown oocytes and assessed the relative level of HIRA and CABIN1, the two other molecules within the HIRA complex. We found that the overall nuclear staining signals of HIRA and CABIN1 were significantly decreased in Ubn1 MO oocytes compared to Ctrl MO oocytes (Supplementary Fig. 2D). This indicated that the loss-of-function of HIRA complex resulted in mislocalisation in the nucleus which suggests the decrease of binding stability. We also performed the Triton X-100 preextraction protocol to remove the unbound chromatinassociated signals in Ubn1 KD oocytes (Nashun et al. 2015), and noted that the level of H3.3 was comparably lowered in these oocytes (Supplementary Fig. 2D).

Similarly, we conducted IF on CabZ KO oocytes. Not only were overall nuclear staining signals of UBN1 and HIRA reduced in CabZ KO oocytes but so was the level of H3.3 (Supplementary Fig. 2E). We also performed the Triton X-100 pre-extraction in CabZ oocytes, and noted that the level of H3.3 was comparably lowered in CabZ KO oocytes (Supplementary Fig. 2E).
The above results suggest that each molecule of HIRA complex is essential for the stability of the complex in the oocyte and loss of any one of them affects male pronucleus formation at fertilisation.

\section{HIRA complex failed to associate with male chromatin of human abnormal 1 PN zygotes}

Based on the promising data collected from mice, we investigated whether the HIRA complex is involved in male pronucleus formation in humans.

Human 1PN embryos were donated for research use after examination of zygotes $18 \mathrm{~h}$ post insemination (following both IVF and ICSI). During the recruitment period of 2018-2019, we approached a total of 218 couples and 144 couples gave consent. Overall, 23 1PN zygotes were identified from 16 IVF/ICSI cycles and 21 were fixed (1PN /fertilised embryo: 21/101=20.1\%; Table 1 and Fig. 3A). Subsequent analysis showed that an additional nine embryos originally identified in the clinic as being $1 \mathrm{PN}$ were at more advanced stages upon collection. Seven had progressed to nuclear envelope breakdown (NEBD) or the 2-cell stage, and two were parthenogenetically activated.

We performed IF on the fixed samples using H3K9me3, a female chromatin specific marker in human (van de Werken et al. 2014), in order to distinguish the origin of the parental chromatin. We identified that there were two 1PN zygotes which were actually morphologically abnormal but cytologically normal diploid zygotes. The parental chromatin had developed asynchronously: the female chromatin had progressed to nuclear envelope breakdown stage, while the male chromatin had remained in the pronuclear stage (Fig. 3B; also observed in Egli et al. (2011)). In these one male PN zygotes, we observed that the HIRA immunostaining signal was enriched (Fig. 3B). In contrast, in the abnormal one female PN zygotes (female chromatin labelled by H3K9me3 (van de Werken et al. 2014)), the male chromatin had failed to form the male pronucleus, remaining in the decondensation stage, and importantly lacking HIRA staining. This is in agreement with the data collected from Hira mutant mice (Fig. 2A).

As in the loss-of-function Ubn 1 fertilised 1PN phenotype in the mouse, we also found that UBN1 was absent in the male chromatin of the 1PN human

Table 1 Summary of the one-pronucleus zygote collection during recruitment period.

\begin{tabular}{|c|c|c|c|c|c|}
\hline \multirow[b]{2}{*}{ Recruitment period } & \multicolumn{3}{|c|}{ Patients, $n$} & \multicolumn{2}{|c|}{ 1PN zygotes, $n$} \\
\hline & Approached & Consented & Showing 1 PN zygotes & Collected & Used $^{+}$ \\
\hline 2018 & 70 & 46 & 8 & 9 & 9 \\
\hline 2019 & 148 & 98 & 8 & 14 & 12 \\
\hline Total & 218 & 144 & 16 & 23 & $21^{*}$ \\
\hline
\end{tabular}

${ }^{+}$Used for experiments; *Not all zygotes were found to be 1PN on assessment in the laboratory, nine were at other stages, specifically one-male pronucleus zygotes as control x2; wrong stages x5, and parthenogenesis x2. 


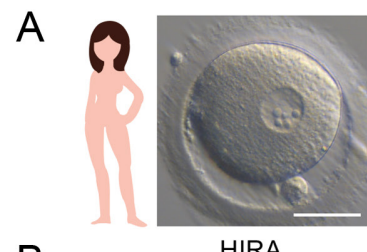

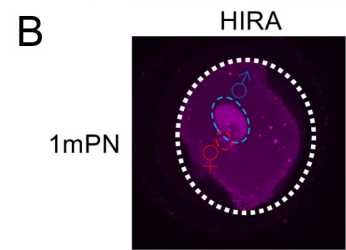

HIRA

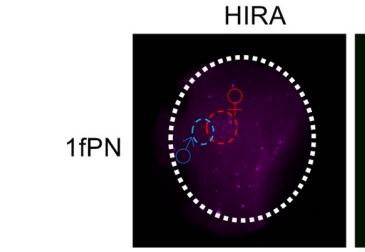

C UBN1

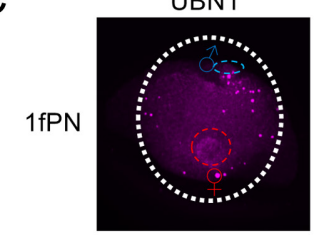

D

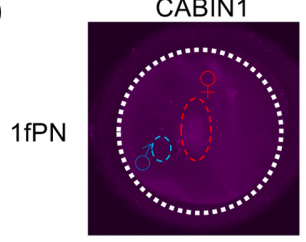

H3K9me3

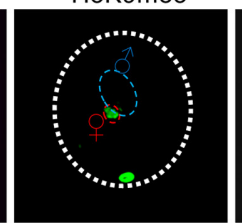
H3K9me3

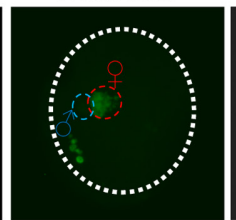

H3K9me3

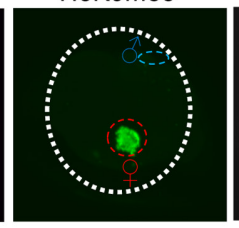

H3K9me3

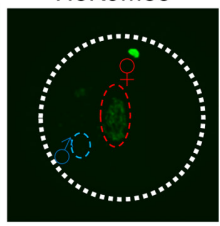

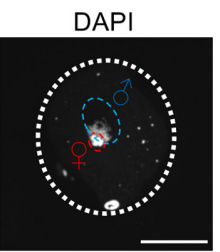

DAPI
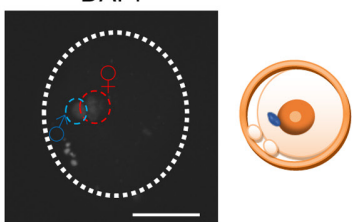

DAPI
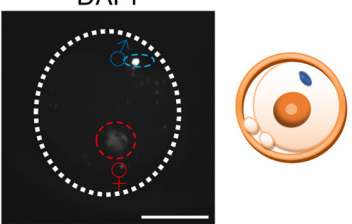

DAPI

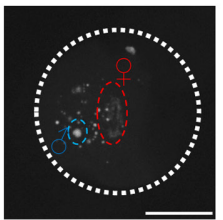

Diagram

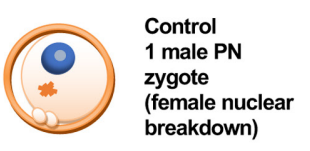

Abnormal

1 female PN

zygote

(failed to form male pronucleus)

Abnormal 1 female PN zygote (failed to form male pronucleus)

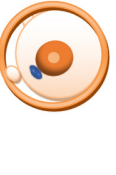

Abnormal

1 female PN zygote male pronucleus) (failed to form
Figure 3 One-pronucleus (1PN) human zygotes revealed deficit of HIRA complex incorporation into the male chromatin. (A) Representative image of a collected human 1PN zygote collected. (B) HIRA failed to associate with the male chromatin of abnormal female $1 \mathrm{PN}$ zygote.

Immunofluorescence of HIRA and H3K9me3 of human $1 \mathrm{PN}$ zygotes. HIRA associates with the male chromatin of a normally fertilised zygote (female chromatin progressed to nuclear breakdown, while male PN remained; upper panel), but failed to associate with the abnormal female $1 \mathrm{PN}$ zygote (failure of male pronucleus formation; lower panel). (C) UBN1 failed to associate with the abnormal 1 female PN zygote. Immunofluorescence of UBN1 and H3K9me3 of a human 1PN zygote. UBN1 failed to associate with the male chromatin. (D) CABIN1 failed to associate with an abnormal 1 female PN zygote. Immunofluorescence of CABIN1 and H3K9me3 of a human 1PN zygote. CABIN1 failed to associate with the male chromatin of an abnormal 1 female PN zygote. Histone H3K9me3 labels female chromatin; DNA counterstained by DAPI. Scale bar $=50 \mu \mathrm{m}$ zygotes (Fig. 3C). We noted that in half of the 1PN zygotes we stained for CABIN1, signal was absent in the male chromatin (Fig. 3D). This was consistent with the abnormal 1PN formation rate in Cabin 1 mutant mice (Fig. 2C). A summary of immunofluorescence results is shown in Table 2.

\section{Overexpression of Hira in mutant oocytes rescues male pronucleus formation in their zygotes}

The results above show that maternal HIRA complex is critical for male pronucleus formation and that loss-

Table 2 Summary of immunofluorescence result of abnormal one-pronucleus zygote.

\begin{tabular}{lccc}
\hline $\begin{array}{l}\text { Stained } \\
\text { antibodies }\end{array}$ & $\begin{array}{c}\text { One fPN zygotes } \\
\text { stained with } \\
\text { antibodies, } \boldsymbol{n}\end{array}$ & $\begin{array}{c}\text { Positive signal } \\
\text { detected in male } \\
\text { chromatin, } \boldsymbol{n}\end{array}$ & Ratio \\
\hline HIRA & 4 & 0 & $0 / 4$ \\
UBN1 & 4 & 0 & $0 / 4$ \\
CABIN1 & 4 & $2^{*}$ & $2 / 4$ \\
Total & 12 & 2 & $2 / 12$ \\
\hline
\end{tabular}

*One with weak signal. of-function leads to abnormal $1 \mathrm{PN}$ phenotypes in mice (Fig. 2). In these abnormal phenotypes, HIRA complex fails to incorporate into male chromatin which is likely to contribute to the abnormal 1 female PN human zygote phenotype (Fig. 3). Both Hira and Cabin 1 null mouse showed later developmental defects than maternal depletion models (see discussion), thus, maternal deposition of HIRA complex has a more profound role than previously understood during the oocyte-toembryo transition, and this cannot be compensated by zygotic transcription rescue during the two-cell stage.

We envisaged that abnormal 1PN zygote phenotypes caused by the maternal defect of Hira could potentially be rescued by overexpression in oocytes. To test this, we first adapted the same micromanipulation platform as we applied to knockdown of Ubn 1 in oocytes (Fig. 4A). Instead of morpholino oligos (Supplementary Fig. $2 \mathrm{~B})$, we injected in vitro transcribed RNA into Hira mutant ( $\mathrm{ZH} \mathrm{KO}$ ) oocytes. The GFP-tagged HIRA protein was stably expressed after culture, and we were able to increase HIRA in the mutant oocytes to a level comparable with that of Hira heterozygous ( $\mathrm{ZH} \mathrm{Het)}$ oocytes ((Supplementary Fig. 3); ZH Het from significant difference of HIRA level of Het vs KO $P=0.023$ to no 


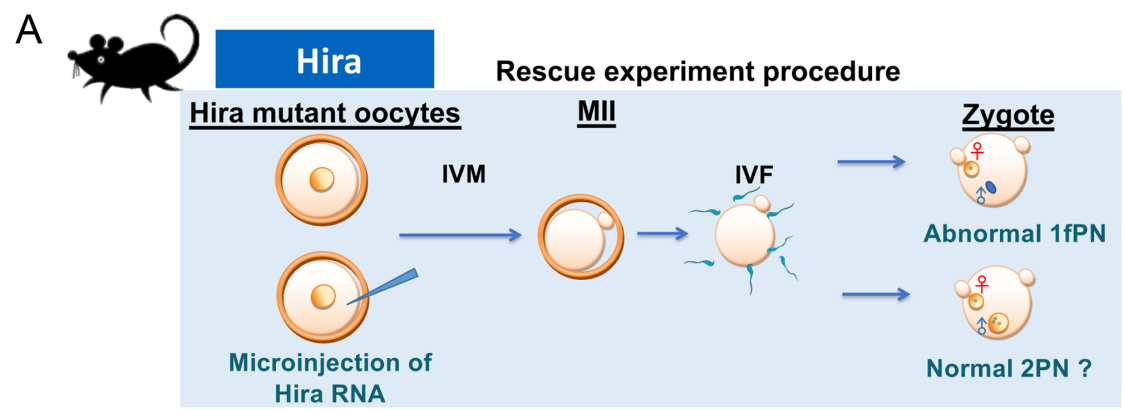

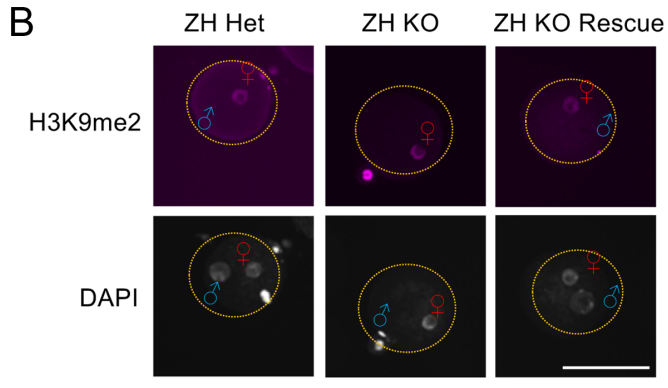

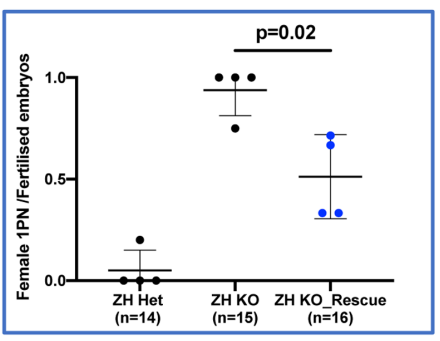

Figure 4 Overexpression of Hira rescued the male pronucleus formation in the Hira mutants. (A) Experimental procedure for rescuing maternal Hira mutants. Hira RNA was introduced into the GV oocytes, following maturation and fertilisation in vitro. Pronuclear formation was examined by

immunofluorescence. (B) Over-expression of Hira RNA in the Hira mutant oocytes rescued the male pronucleus formation after fertilisation. Immunofluorescence of H3K9me2 showed that zygote with two pronuclei was formed after rescue (left panel). Summary of the rescue experiments (right panel). Scale bar $=80 \mu \mathrm{m}$. significance of Het vs KO-injected). On the other hand, the level of $\mathrm{H} 3.3$ showed no significant change which indicated that HIRA overexpression does not alter overall H3.3 expression, while still enabling its histone chaperoning ability of loading H3.3 incorporation into chromatin.

We then introduced Hira RNA into Hira mutant oocytes. In vitro maturation was conducted, and mature oocytes were next fertilised in vitro. To facilitate the fertilisation process not hampered by zona hardening during prolonged culture and address whether penetrated sperm heads can properly decondense (Lin et al. 2014), we removed zona pellucida before in vitro fertilisation. We determined formation of the male pronucleus as an indicator of a successful rescue event by IF (Fig. 4B). $\mathrm{ZH} \mathrm{KO}$ fertilised embryos showed a high proportion of abnormal one female PN zygotes compared to $\mathrm{ZH}$ Het zygotes $(86.7 \%$ vs $7 \%, P=2.7 \mathrm{E}-17)$. However, in $\mathrm{ZH}$ KO oocytes with Hira RNA injection partially rescued the proportion of abnormal one female PN zygotes from 86.7 to $56 \%$ ( $P=0.02$; Fig. $4 \mathrm{~B})$. This result clearly supports our assertion that overexpression of HIRA in null oocytes can support zygote formation, thus providing further support for the proof-of-principle approach to rescuing poor quality oocytes (here, null Hira) in order to support oocyte-to-embryo transition.

\section{Discussion}

We have demonstrated, that in mice, Cabin 1 and Ubn 1 are maternal factors which are associated with decondensed male chromatin after fertilisation and physically interact with HIRA. We showed that molecules within the HIRA complex, CABIN1 (a mammalian specific H3.3 chaperone (Orsi et al. 2013)) and UBN1, acting interdependently, are also critical for male pronucleus formation in mouse. The roles of $\mathrm{H} 3.3$ and HIRA have been reported in bovine pre-implantation stages (Zhang et al. 2016,2018), but relevant study, particularly focusing on maternal roles, has been lacking. Improved understanding will be beneficial for the improvement of domestic animal production. Importantly, our investigation of abnormal human 1PN zygotes showed that HIRA complex was absent in the male chromatin, thus linking this unique phenotype with a potentially coherent mechanism of zygote formation in mice and humans. Using our mouse model, we showed that we could ameliorate the deleterious effects of the loss of Hira, thus providing proof of concept of rescue as a potential therapeutic approach.

Results of molecular cytogenetics of human 1PN zygotes have been reported. These results show that the possible underlying causes include synchronous PN formation, fusion of maternal and paternal PN, parthenogenetic activation, or premature breakdown of PN (Azevedo et al. 2014). In our limited but carefully phenotyped $1 \mathrm{PN}$ samples $(n=21)$ we observed that the majority showed failure of male pronucleus formation (Fig. 3), indicating an abnormal fertilisation event. Other zygotes, initially identified as having $1 \mathrm{PN}$, were subsequently shown to have asynchronous PN formation $(n=2$; as the IF controls), parthenogenetic activation $(n=2)$, or incorrect staging (progressed to nuclear breakdown and two cells; $n=5$ ) (Table 1 ). Our results support the statement that, in general, $1 \mathrm{PN}$ zygotes should be considered to have undergone abnormal fertilisation and to be less competent of progressing to a normal embryo, (Yao et al. 2016, Araki et al. 2018, Destouni et al. 2018).

Originally, null mutants demonstrated the requirement for Hira around the time of gastrulation (Roberts et al. 2002) but our previous studies discovered that maternal 
Hira is essential for zygote development (Lin et al. 2014, Nashun et al. 2015). Similarly, Cabin 1 null mice showed embryo lethality around embryonic day 12.5 due to organogenesis defects (Esau et al. 2001). Interestingly, our oocyte-deleted Cabin 1 mutants revealed preimplantation defects (Smith et al. 2020 bioRxiv). Based on the results of our Hira maternal mouse models, which revealed a great loss of developmental propensity (Lin et al. 2014), we envisaged that the lower developmental potential of human 1PN embryos might be due to defects of embryonic genome activation, arising at the $4-8$ cell stages in humans. It would be valuable to measure the transcriptome of abnormal $1 \mathrm{PN}$ zygotes to establish a profile of the downstream effectors as a 'good oocyte' signature. Also, it will be valuable to monitor the subsequent pregnancy outcomes from patients who donated the 1PN zygotes and the reoccurrence of $1 \mathrm{PN}$ zygotes after new cycles.

Next, we mined the human exon sequence dataset, GenomeAD Browser (https://gnomad.broadinstitute. org), to examine whether there is a genetic linkage relating to the loss-of-function of HIRA complex. We noted that the loss-of-function observed/expected upper bound fraction (LOEUF) parameters of HIRA (0.02-0.14) and UBN1 (0.05-0.21) are very low (CABIN1 (0.36-0.59)), indicating low tolerance to inactivation of the genes. In other words, both HIRA and UBN1 likely tend to result in loss-of-function mutations. The interdependence of HIRA complex components shown in Supplementary Fig. 2D and E, combining the results from Nashun et al. (Nashun et al. 2015) and the previous data in human cells (Rai et al. 2011) indicate that the normal function of all three components of HIRA complex is required for its adequate function. Similarly, the abnormal human $1 \mathrm{PN}$ zygotes we collected were possibly inherited from loss-of-function HIRA complex oocytes. This means that abnormal $1 \mathrm{PN}$ zygotes might harbour divergent transcriptomic and chromatin profiles. Therefore, it will be of interest to collect human 1PN zygotes and perform RNA-sequencing or chromatin-associated assays (ATACseq (Wu et al. 2016) and Hi-C (Flyamer et al. 2017) or scNMT-seq (Clark et al. 2018)) to gain insight into human paternal genome reprogramming events.

Overexpression of Hira mRNA in mutant murine oocytes partially rescued the abnormal $1 \mathrm{PN}$ phenotype by significantly restoring mutant oocyte fertilisation to form normal two PN zygotes (Fig. 4). This not only demonstrated that maternally stored HIRA is critical for male pronucleus formation but also provided a proof-ofprinciple experiment as the foundation of an approach for rescuing defects caused by impaired maternal factors. A rescue attempt of Hira 1PN zygote phenotype has been showed by co-injection of Hira siRNA with mRNA during oogenesis (Inoue \& Zhang 2014). Our new rescue result reinforces the applicability of this approach. We introduced Hira mRNA into fully grown
Hira mutant GV oocytes, the end stage of oogenesis, and partially restored the abnormal $1 \mathrm{PN}$ zygote phenotype to normal phenotype. Since Zp3-Cre driven recombination happens during oogenesis, both maternal Hira mRNA and protein have been depleted. The consequent feasibility of rescuing mutant oocytes at an advanced oocyte stage directly demonstrates the expansion of the window of opportunity to rescue defective maternal effect genes. Also, fully grown GV oocytes are the ideal stage for oocyte manipulation compared to growing oocytes.

Besides the likelihood of the HIRA-mediated H3.3 incorporation mechanism being directly involved in paternal chromatin assembly, Gou et al. (2020) reported that Srpk1, a splicing kinase, not only acts as a key factor in phosphorylating protamine to initiate paternal genome programming but it also facilitates protamine interaction with HIRA for $\mathrm{H} 3.3$ deposition. It will be worth investigating whether CABIN1 or UBN1 is associated with SRPK1, and whether SRPK1 is the upstream regulator to initiate the nucleosome assembly process. Another possible avenue would be to examine the overall transcriptional impact of HIRA complex in the mutant oocytes, in order to investigate whether there is an effect on deposition of maternal factors during oogenesis (e.g. Srpk1 or others). On the other hand, careful dissection of the downstream consequences of absence of HIRA complex, such as profiling the global chromatin landscape by genome-wide approaches, examining the formation of nuclear pore envelope assembly after failure of nucleosome assembly (Inoue \& Zhang 2014) or further determining the transcriptional impact, require further endeavour. These future studies will not only lead towards a better understanding of the mechanism of action but also advance future clinical diagnosis and identification of potential biomarkers of the $1 \mathrm{PN}$ phenotype in the human.

In conclusion, by generating new loss-of-function mouse models and analysing human $1 \mathrm{PN}$ zygotes, we report new molecular insights into human 1PN zygote formation.

\section{Supplementary materials}

This is linked to the online version of the paper at https://doi. org/10.1530/REP-20-0636.

\section{Declaration of interest}

The authors declare that there is no conflict of interest that could be perceived as prejudicing the impartiality of the research reported.

\section{Funding}

This work was supported by MRC Centre Grant MR/ N022556/1, the Wellcome Trust-University of Edinburgh 
Institutional Strategic Support Fund, Barbour Watson Trust, and grants from Deanery of Clinical Sciences, College of Medicine \& Veterinary Medicine of University of Edinburgh to C-J L. C-J $\mathrm{L}$ is a Royal Society of Edinburgh Personal Research Fellow funded by the Scottish Government.

\section{Author contribution statement}

$\mathrm{R} S$ involved in mouse colony management and performed oocyte experiments. S J P and A K performed human IVF treatment under the overall management of $\mathrm{KJ}$ T. R A A is a HFEA licence holder. C-J L, R S, and R A A interpreted the data. C-J $L$ conceived the project and designed the experiments, performed all embryo micromanipulation and human IF work. R S, R A A, and C-J L wrote the manuscript with input from all authors.

\section{Acknowledgements}

The authors thank Anne Saunderson and Isobel Morton for recruitment of patients and management the clinical information. The authors also thank Martin Anger for the plasmid of GFP (McGuinness et al. 2009), Debasree Dutta for the plasmid of GFP-Hira (Majumder et al. 2015), and Peter Adams for the antibody of UBN1. The authors appreciate Profs Andrew Horne and Norah Spears for their constructive comments.

\section{References}

Araki E, Itoi F, Honnma H, Asano Y, Oguri H \& Nishikawa K 2018 Correlation between the pronucleus size and the potential for human single pronucleus zygotes to develop into blastocysts : 1PN zygotes with large pronuclei can expect an embryo development to the blastocyst stage that is similar to the development of $2 \mathrm{PN}$ zygotes. Journal of Assisted Reproduction and Genetics 35 817-823. (https://doi. org/10.1007/s10815-018-1137-1)

Azevedo AR, Pinho MJ, Silva J, Sa R, Thorsteinsdottir S, Barros A \& Sousa M 2014 Molecular cytogenetics of human single pronucleated zygotes. Reproductive Sciences 21 1472-1482. (https://doi. org/10.1177/1933719114530185)

Buschbeck M \& Hake SB 2017 Variants of core histones and their roles in cell fate decisions, development and cancer. Nature Reviews: Molecular Cell Biology 18 299-314. (https://doi.org/10.1038/nrm.2016.166)

Chen J, Torcia S, Xie F, Lin CJ, Cakmak H, Franciosi F, Horner K, Onodera C, Song JS, Cedars MI et al. 2013 Somatic cells regulate maternal mRNA translation and developmental competence of mouse oocytes. Nature Cell Biology 15 1415-1423. (https://doi.org/10.1038/ncb2873)

Clark SJ, Argelaguet R, Kapourani CA, Stubbs TM, Lee HJ, Alda-Catalinas C, Krueger F, Sanguinetti G, Kelsey G, Marioni JC et al. 2018 scNMT-seq enables joint profiling of chromatin accessibility DNA methylation and transcription in single cells. Nature Communications 9 781. (https://doi. org/10.1038/s41467-018-03149-4)

De Vries WN, Binns LT, Fancher KS, Dean J, Moore R, Kemler R \& Knowles BB 2000 Expression of Cre recombinase in mouse oocytes: a means to study maternal effect genes. Genesis 26 110-112. (https://doi.org/10.1002/(SICl)1526-968X(200002)26:2<110::AIDGENE2>3.0.CO;2-8)

Destouni A, Dimitriadou E, Masset H, Debrock S, Melotte C, Van Den Bogaert K, Esteki MZ, Ding J, Voet T, Denayer E et al. 2018 Genomewide haplotyping embryos developing from OPN and 1PN zygotes increases transferrable embryos in PGT-M. Human Reproduction 33 2302-2311. (https://doi.org/10.1093/humrep/dey325)

Egli D, Chen AE, Saphier G, Ichida J, Fitzgerald C, Go KJ, Acevedo N, Patel J, Baetscher M, Kearns WG et al. 2011 Reprogramming within hours following nuclear transfer into mouse but not human zygotes. Nature Communications 2 488. (https://doi.org/10.1038/ncomms1503)

Esau C, Boes M, Youn HD, Tatterson L, Liu JO \& Chen J 2001 Deletion of calcineurin and myocyte enhancer factor 2 (MEF2) binding domain of Cabin 1 results in enhanced cytokine gene expression in T cells. Journal of Experimental Medicine 194 1449-1459. (https://doi.org/10.1084/ jem.194.10.1449)

Flyamer IM, Gassler J, Imakaev M, Brandao HB, Ulianov SV, Abdennur N, Razin SV, Mirny LA \& Tachibana-Konwalski K 2017 Single-nucleus Hi-C reveals unique chromatin reorganization at oocyte-to-zygote transition. Nature 544 110-114. (https://doi.org/10.1038/nature21711)

Gosden R 1996 Reproductive biology. The vocabulary of the egg. Nature 383 485-486. (https://doi.org/10.1038/383485a0)

Gou LT, Lim DH, Ma W, Aubol BE, Hao Y, Wang X, Zhao J, Liang Z, Shao C, Zhang X et al. 2020 Initiation of parental genome reprogramming in fertilized oocyte by splicing kinase SRPK1-catalyzed protamine phosphorylation. Cell 180 1212.e14-1227.e14. (https://doi. org/10.1016/j.cell.2020.02.020)

Hammond CM, Stromme CB, Huang H, Patel DJ \& Groth A 2017 Histone chaperone networks shaping chromatin function. Nature Reviews: Molecular Cell Biology 18 141-158. (https://doi.org/10.1038/ nrm.2016.159)

Hasegawa A, Mochida K, Inoue H, Noda Y, Endo T, Watanabe G \& Ogura A 2016 High-yield superovulation in adult mice by anti-inhibin serum treatment combined with estrous cycle synchronization. Biology of Reproduction 94 21. (https://doi.org/10.1095/ biolreprod.115.134023)

Inoue A \& Zhang Y 2014 Nucleosome assembly is required for nuclear pore complex assembly in mouse zygotes. Nature Structural and Molecular Biology 21 609-616. (https://doi.org/10.1038/nsmb.2839)

Lin CJ, Conti M \& Ramalho-Santos M 2013 Histone variant H3.3 maintains a decondensed chromatin state essential for mouse preimplantation development. Development 140 3624-3634. (https://doi.org/10.1242/ dev.095513)

Lin CJ, Koh FM, Wong P, Conti M \& Ramalho-Santos M 2014 Hira-mediated $\mathrm{H} 3.3$ incorporation is required for DNA replication and ribosomal RNA transcription in the mouse zygote. Developmental Cell 30 268-279. (https://doi.org/10.1016/j.devcel.2014.06.022)

Loppin B, Bonnefoy E, Anselme C, Laurencon A, Karr TL \& Couble P 2005 The histone H3.3 chaperone HIRA is essential for chromatin assembly in the male pronucleus. Nature 437 1386-1390. (https://doi.org/10.1038/ nature04059)

Majumder A, Syed KM, Joseph S, Scambler PJ \& Dutta D 2015 Histone chaperone HIRA in regulation of transcription factor RUNX1. Journal of Biological Chemistry 290 13053-13063. (https://doi.org/10.1074/jbc. M114.615492)

Maze I, Noh KM, Soshnev AA \& Allis CD 2014 Every amino acid matters: essential contributions of histone variants to mammalian development and disease. Nature Reviews: Genetics 15 259-271. (https://doi. org/10.1038/nrg3673)

McGuinness BE, Anger M, Kouznetsova A, Gil-Bernabe AM, Helmhart W, Kudo NR, Wuensche A, Taylor S, Hoog C, Novak B et al. 2009 Regulation of APC/C activity in oocytes by a Bub1-dependent spindle assembly checkpoint. Current Biology 19 369-380. (https://doi.org/10.1016/j. cub.2009.01.064)

Nashun B, Hill PW, Smallwood SA, Dharmalingam G, Amouroux R, Clark SJ, Sharma V, Ndjetehe E, Pelczar P, Festenstein RJ et al. 2015 Continuous histone replacement by Hira is essential for normal transcriptional regulation and de novo DNA methylation during mouse oogenesis. Molecular Cell 60 611-625. (https://doi.org/10.1016/j. molcel.2015.10.010)

Orsi GA, Algazeery A, Meyer RE, Capri M, Sapey-Triomphe LM, Horard B, Gruffat H, Couble P, Ait-Ahmed O \& Loppin B 2013 Drosophila Yemanuclein and Hira cooperate for de novo assembly of H3.3containing nucleosomes in the male pronucleus. PLoS Genetics 9 e1003285. (https://doi.org/10.1371/journal.pgen.1003285)

Paull D, Emmanuele V, Weiss KA, Treff N, Stewart L, Hua H, Zimmer M, Kahler DJ, Goland RS, Noggle SA et al. 2013 Nuclear genome transfer in human oocytes eliminates mitochondrial DNA variants. Nature 493 632-637. (https://doi.org/10.1038/nature11800)

Percharde M, Lin CJ, Yin Y, Guan J, Peixoto GA, Bulut-Karslioglu A, Biechele S, Huang B, Shen X \& Ramalho-Santos M 2018. A Line 1- 
Nucleolin Partnership regulates early development and ESC identity. Cell 174 391-405. (https://doi.org/10.1016/j.cell.2018.05.043)

Rai TS, Puri A, Mcbryan T, Hoffman J, Tang Y, Pchelintsev NA, Van Tuyn J, Marmorstein R, Schultz DC \& Adams PD 2011 Human CABIN1 is a functional member of the human HIRA/UBN1/ASF1a histone H3.3 chaperone complex. Molecular and Cellular Biology 31 4107-4118. (https://doi.org/10.1128/MCB.05546-11)

Ray-Gallet D, Ricketts MD, Sato Y, Gupta K, Boyarchuk E, Senda T, Marmorstein R \& Almouzni G 2018 Functional activity of the H3.3 histone chaperone complex HIRA requires trimerization of the HIRA subunit. Nature Communications 9 3103. (https://doi.org/10.1038/ s41467-018-05581-y)

Ricketts MD, Frederick B, Hoff H, Tang Y, Schultz DC, Singh Rai T, Grazia Vizioli M, Adams PD \& Marmorstein R 2015 Ubinuclein-1 confers histone $\mathrm{H} 3.3$-specific-binding by the HIRA histone chaperone complex. Nature Communications 6 7711. (https://doi.org/10.1038/ncomms8711)

Roberts C, Sutherland HF, Farmer H, Kimber W, Halford S, Carey A, Brickman JM, Wynshaw-Boris A \& Scambler PJ 2002 Targeted mutagenesis of the Hira gene results in gastrulation defects and patterning abnormalities of mesoendodermal derivatives prior to early embryonic lethality. Molecular and Cellular Biology 22 2318-2328. (https://doi.org/10.1128/mcb.22.7.2318-2328.2002)

Sciorio R, Thong JK \& Pickering SJ 2018 Comparison of the development of human embryos cultured in either an embryoscope or benchtop incubator. Journal of Assisted Reproduction and Genetics 35 515-522. (https://doi.org/10.1007/s10815-017-1100-6)

Si J, Zhu X, Lyu Q \& Kuang Y 2019 Obstetrical and neonatal outcomes after transfer of cleavage-stage and blastocyst-stage embryos derived from monopronuclear zygotes: a retrospective cohort study. Fertility and Sterility 112 527-533. (https://doi.org/10.1016/j.fertnstert.2019.04.045)

Smith R, Pickering S, Kopakaki A, Thong KJ, Anderson RA \& C-J Lin 2020 Histone H3.3 Hira chaperone complex contributes to zygote formation in mice and humans. bioRxiv (https://doi.org/10.1101/2020.06.18.159954)

Swain JE \& Pool TB 2008 ART failure: oocyte contributions to unsuccessful fertilization. Human Reproduction Update 14 431-446. (https://doi. org/10.1093/humupd/dmn025)

Sztein JM, Farley JS \& Mobraaten LE 2000 In vitro fertilization with cryopreserved inbred mouse sperm. Biology of Reproduction 63 1774-1780. (https://doi.org/10.1095/biolreprod63.6.1774)
Tang MC, Jacobs SA, Mattiske DM, Soh YM, Graham AN, Tran A, Lim SL, Hudson DF, Kalitsis P, O'bryan MK et al. 2015 Contribution of the two genes encoding histone variant $\mathrm{h} 3.3$ to viability and fertility in mice. PLoS Genetics 11 e1004964. (https://doi.org/10.1371/journal.pgen.1004964)

Van De Werken C, Van Der Heijden GW, Eleveld C, Teeuwssen M, Albert M, Baarends WM, Laven JS, Peters AH \& Baart EB 2014 Paternal heterochromatin formation in human embryos is H3K9/HP1 directed and primed by sperm-derived histone modifications. Nature Communications 5 5868. (https://doi.org/10.1038/ncomms6868)

Wu J, Huang B, Chen H, Yin Q, Liu Y, Xiang Y, Zhang B, Liu B, Wang Q, Xia W et al. 2016 The landscape of accessible chromatin in mammalian preimplantation embryos. Nature $\mathbf{5 3 4}$ 652-657. (https://doi.org/10.1038/ nature18606)

Yao G, Xu J, Xin Z, Niu W, Shi S, Jin H, Song W, Wang E, Yang Q, Chen L et al. 2016 Developmental potential of clinically discarded human embryos and associated chromosomal analysis. Scientific Reports 6 23995. (https://doi.org/10.1038/srep23995)

Zhang K, Rajput SK, Wang S, Folger JK, Knott JG \& Smith GW 2016 $\mathrm{CHD} 1$ regulates deposition of histone variant $\mathrm{H} 3.3$ during bovine early embryonic development. Biology of Reproduction 94 140. (https://doi. org/10.1095/biolreprod.116.138693)

Zhang K, Wang H, Rajput SK, Folger JK \& Smith GW 2018 Characterization of $\mathrm{H} 3.3$ and HIRA expression and function in bovine early embryos. Molecular Reproduction and Development 85 106-116. (https://doi. org/10.1002/mrd.22939)

Zhao ZK, Li W, Wang MY, Zhou L, Wang JL \& Wang YF 2011 The role of HIRA and maternal histones in sperm nucleus decondensation in the gibel carp and color crucian carp. Molecular Reproduction and Development 78 139-147. (https://doi.org/10.1002/mrd.21278)

Received 1 December 2020

First decision 23 December 2020

Revised Manuscript received 24 March 2021

Accepted 8 April 2021 